\title{
CLASSES OF WIRING DIAGRAMS AND THEIR INVARIANTS
}

\author{
DAVID GARBER ${ }^{1}$, MINA TEICHER ${ }^{1}$ AND UZI VISHNE ${ }^{2}$
}

\begin{abstract}
Wiring diagrams usually serve as a tool in the study of arrangements of lines and pseudolines. Here we go in the opposite direction, using known properties of line arrangements to motivate certain equivalence relations and actions on sets of wiring diagrams, which preserve the incidence lattice and the fundamental groups of the affine and projective complements of the diagrams.

These actions are used in GTV to classify real arrangements of up to 8 lines and show that in this case, the incidence lattice determines both the affine and the projective fundamental groups.
\end{abstract}

\section{INTRODUCTION}

A line arrangement in $\mathbb{C}^{2}$ is a union of finitely many copies of $\mathbb{C}^{1}$. An arrangement is called real if the defining equations of its lines can be written with real coefficients, and complex otherwise.

The fundamental groups of the complement in $\mathbb{C P}^{2}$ and of its affine part in $\mathbb{C}^{2}$ are called the projective and affine fundamental groups of the arrangement, respectively.

A more combinatorial invariant is the incidence lattice of the arrangement, which consists of the lines, their intersection points, the empty set and $\mathbb{C P}^{2}$, ordered by inclusion.

To a real arrangement, one can associate a combinatorial object, called a wiring diagram. This is not an invariant, as it depends on the choice of a guiding generic line. Still, this object turns out to be a useful tool in the study of arrangements of lines, $c f$. [MoTe], [GTV] or CS.

In this paper, we show how the natural actions on the plane induce actions on sets of wiring diagrams. Likewise, identities in the braid

\footnotetext{
${ }^{1}$ Partially supported by The Israel Science Foundation (Center of Excellence Program), by the Emmy Noether Institute for Mathematics and by the Minerva Foundation (Germany). The research was done during the Ph.D. studies of David Garber, under the supervision of Prof. Mina Teicher.

${ }^{2}$ Partially supported by the Edmund Landau Center for Research in Mathematical Analysis and Related Subjects.

Date: November 11, 2018.
} 
group induce some equivalence relations on these sets. The actions and relations will be shown to preserve the incidence lattice and the affine and projective fundamental groups. We also study their relations and show that they induce dihedral group actions on the set of diagrams.

In general, there are millions of wiring diagrams of up to 8 lines, each equipped with its own fundamental groups. In GTV we use the actions and relations intorduced here to show that there are at most 103 distinct fundamental groups, and they are determined by the incidence lattice of the diagram.

The paper is organized as follows. In section 2 we present some combinatorial objects which are associated to real wiring diagrams: the list of Lefschetz pairs, the incidence lattice, and the signature. Section 3 describes the algorithm commonly used for computing finite presentations of the affine and projective fundamental groups.

In section 1 we define and study equivalence relations on the set of wiring diagrams, and discuss certain actions on the equivalence classes. We prove that these relations and actions preserve the incidence lattice and the affine and projective fundamental groups. Finally, in section 5 we discuss some connections between the various actions, and a numerical example. The focus in this paper is on the actions and relations, and their properties. The application to classification of arrangements with up to 8 real lines is given in GTV.

\section{Combinatorial PREliminaries}

We briefly recall some of the combinatorial objects and constructions related to arrangements of lines.

2.1. Wiring diagrams and Lefschetz pairs. Given a real arrangement of lines in $\mathbb{C}^{2}$, the intersection of its affine part with the natural copy of $\mathbb{R}^{2}$ in $\mathbb{C}^{2}$ is an arrangement of lines in the real plane.

To an arrangement of $\ell$ lines in $\mathbb{R}^{2}$ one can associate a wiring diagram Go, which holds the combinatorial data of the arrangement and the position of the intersection points. A wiring diagram is a collection of $\ell$ wires (where a wire in $\mathbb{R}^{2}$ is a union of segments and rays, homeomorphic to $\mathbb{R}$ ). The induced wiring diagram is constructed by choosing a new line (called the guiding line), which avoids all the intersection points of the arrangement, such that the projections of intersection points do not overlap. Then, $\ell$ wires are generated as follows. Start at the ' $\infty$ ' end of the line with $\ell$ parallel rays, and for every projection of an intersection point, make the corresponding switch in the rays, as in Figure 1 . 


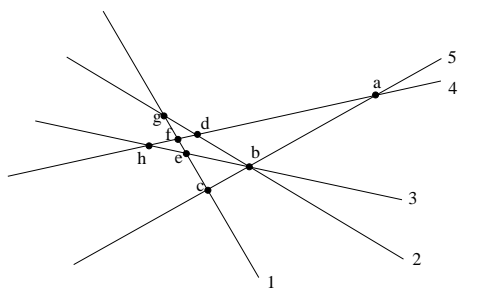

guiding line

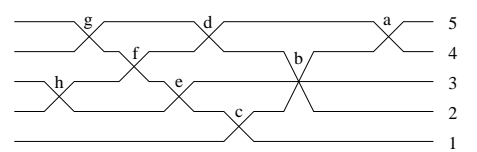

\section{FiguRE 1.}

To a wiring diagram, one can associate a list of Lefschetz pairs. Any pair of this list corresponds to one of the intersection points, and holds the smallest and the largest indices of the wires intersected at this point, numerated locally near the intersection point (see MoTe and GaTe ).

For example, in the wiring diagram of Figure 2,

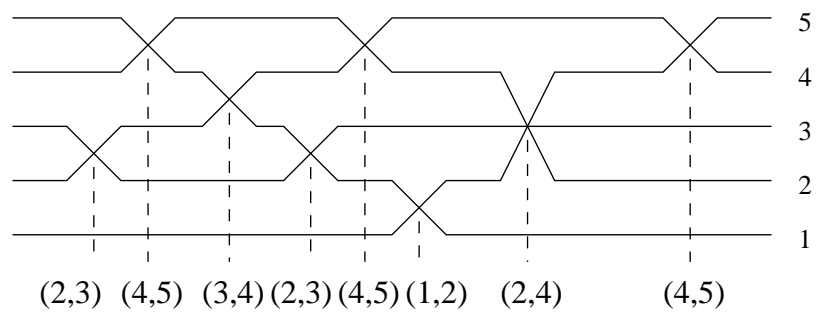

Figure 2.

the list of Lefschetz pairs is:

$$
([4,5],[2,4],[1,2],[4,5],[2,3],[3,4],[4,5],[2,3]) .
$$

To every list of Lefschetz pairs there is a corresponding wiring diagram, which is constructed by the reverse procedure.

At some points in this paper it would be necessary to slightly change the definition of a wiring diagram, by fixing some angles $\alpha_{1}<\cdots<\alpha_{\ell}$, and assuming that the $i$ th wire is 'broken' at some distant positive point at angle $\alpha_{i}$, and at some distant negative point at angle $-\alpha_{i}$; all with respect to the guiding line. This is done to prevent the wires from meeting at infinity; whenever we discuss the projective fundamental group, the diagrams are meant to have this structure. 
2.2. The incidence lattice of an arrangement. Let $\mathcal{L}=\left\{L_{1}, \cdots, L_{\ell}\right\}$ be an arrangement of lines. By $\operatorname{Lat}(\mathcal{L})$ we denote the partially-ordered set of non-empty intersections of the $L_{i}$, ordered by inclusion (see OrT]). We include the whole plane and the empty set in $\operatorname{Lat}(\mathcal{L})$, so that it becomes a lattice.

For example, if we numerate the intersection points of the arrangement of lines in Figure 1, as presented in Figure 3,

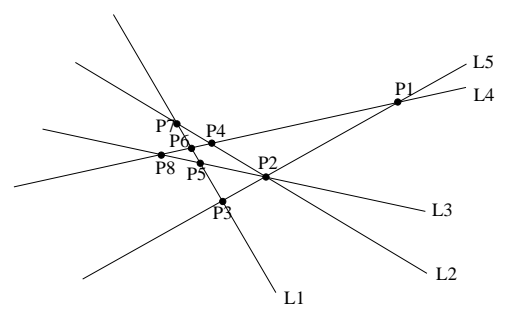

\section{FiguRE 3.}

we get the lattice presented in Figure 4 .

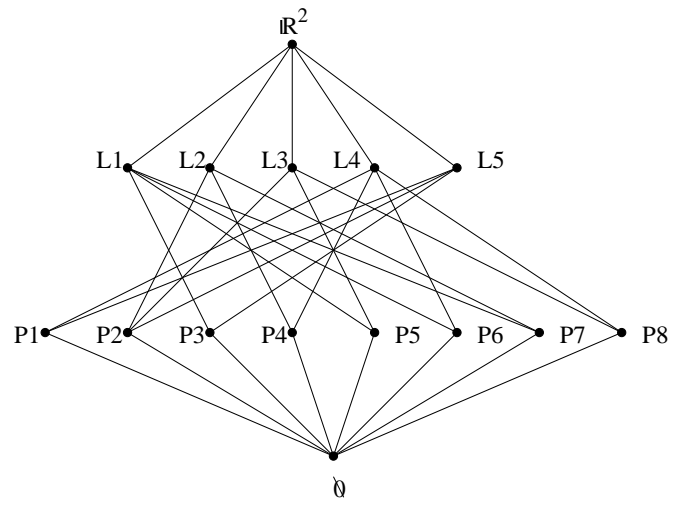

FIGURE 4.

2.3. The signature. We define another combinatorial invariant of line arrangements: the signature.

Definition 2.1. The signature of an arrangement is $\left[2^{n_{2}} 3^{n_{3}} \cdots\right]$ where $n_{k}$ is the number of points in which $k$ lines intersect.

We make the agreement to omit every $c^{0}$.

Obviously, any wiring diagram induced by an arrangement of lines has the property that every two wires intersect each other exactly once. This is the unique intersection property. 
Remark 2.2. Note that from the unique intersection property it follows that the order of the lines in $-\infty$ is the inverse of that in $\infty$.

An easy property for the signature $\left[2^{n_{2}} 3^{n_{3}} \ldots\right]$ of a wiring diagram with $\ell$ lines is that

$$
\sum_{k \geq 2} n_{k}\left(\begin{array}{l}
k \\
2
\end{array}\right)=\left(\begin{array}{l}
\ell \\
2
\end{array}\right)
$$

(there are $\left(\begin{array}{l}\ell \\ 2\end{array}\right)$ simple intersection points in a generic line arrangement with $\ell$ lines, and every point of multiplicity $k$ replaces $\left(\begin{array}{l}k \\ 2\end{array}\right)$ simple intersection points).

\section{Computation of the fundamental Group}

In this section we present the computation of a the fundamental groups of the complement of (complexified) wiring diagram. This is an easy generalization of the Moishezon-Teicher method [MoTe and the van Kampen theorem, which are usually used to compute the fundamental group of the complement of line arrangements. The algorithm is used in the proof of Theorems 4.11 and 4.13 below. A proof for the algorithm itself can be found in MoTe.

Let $D$ be a closed disk in $\mathbb{R}^{2}, K \subset \operatorname{Int}(D)$ a set of $\ell$ points, and $u \in \partial D$. Let $\mathcal{B}$ be the group of all diffeomorphisms $\beta: D \rightarrow D$ such that $\left.\beta\right|_{\partial D}$ is the identity and $\beta(K)=K$. The action of such $\beta$ on the disk applies to paths in $D$, which induces an automorphism on $\pi_{1}(D-K, u)$. The braid group, $B_{\ell}[D, K]$, is the group $\mathcal{B}$ modulo the subgroup of diffeomorphisms inducing the trivial automorphism on $\pi_{1}(D-K, u)$. An element of $B_{\ell}[D, K]$ is called a braid.

Let $\sigma \subset \operatorname{Int}(D)$ be a path connecting two points $a, b \in K$, which avoids all the other points in $K$. Using $\sigma$ one defines a diffeomorphism of $D$ by exchanging $a, b$ along the path (more precisely, along two paths parallel to $\sigma$ ). The resulting braid is called a half-twist, and denoted by $H(\sigma)$. It can be seen that $B_{\ell}[D, K]$ is generated by the half-twists. For simplicity, we will assume that $D=\left\{z \in \mathbb{C}:\left|z-\frac{\ell+1}{2}\right| \leq \frac{\ell+1}{2}\right\}$, and that $K=\{1,2, \cdots, \ell\} \subset D$.

Choose a point $u_{0} \in D$ (for convenience we choose it to be below the real line). The group $\pi_{1}\left(D-K, u_{0}\right)$ is freely generated by $\Gamma_{1}, \ldots, \Gamma_{\ell}$, where $\Gamma_{i}$ is a loop starting and ending at $u_{0}$, enveloping the $i$ th point in $K$. The set $\left\{\Gamma_{1}, \ldots, \Gamma_{\ell}\right\}$ is called a geometric base or $g$-base of $\pi_{1}\left(\mathbb{C}^{2}-\right.$ $\left.K, u_{0}\right)$.

Let $\left(\left[a_{1}, b_{1}\right], \ldots,\left[a_{p}, b_{p}\right]\right)$ be a list of Lefschetz pairs associated to a wiring diagram $\mathbf{w}$ with $\ell$ wires. The fundamental group of the complement of the diagram is a quotient group of $\pi_{1}\left(D-K, u_{0}\right)$. There are $p$ 
relations, one for every intersection point. In each point we will compute an object called a skeleton, from which the relation is computed.

Let $\mathbf{a}=\left(\left[a_{1}, b_{1}\right], \cdots,\left[a_{p}, b_{p}\right]\right)$ be a list of Lefschetz pairs. In order to compute the skeleton $s_{i}$ associated to the $i$ th intersection point $\left[a_{i}, b_{i}\right]$ we start with an initial skeleton which is presented in Figure 5, in which the points correspond to the lines of the corresponding wiring diagram.

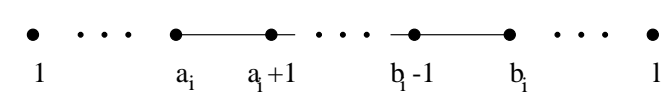

\section{Figure 5 .}

To this skeleton we apply the Lefschetz pairs $\left[a_{i-1}, b_{i-1}\right], \cdots,\left[a_{1}, b_{1}\right]$. A Lefschetz pair $\left[a_{j}, b_{j}\right]$ acts by rotating the region from $a_{j}$ to $b_{j}$ by $180^{\circ}$ counterclockwise without affecting any other points.

For example, consider the list $\mathbf{a}=([2,3],[2,4],[4,5],[1,3],[3,4])$. The initial skeleton for $[3,4]$ is given in Figure 6 .

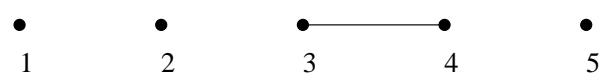

Figure 6.

Applying [1,3] and then $[4,5]$, we get the skeleton of Figure 7 .

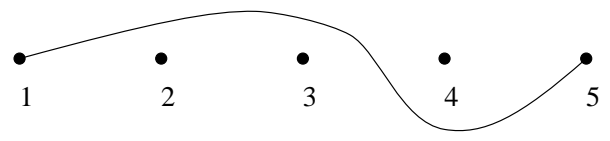

FiguRE 7.

Then, applying $[2,4]$ yields the skeleton of Figure 8, and finally acting with $[2,3]$ we get the skeleton in Figure 9.

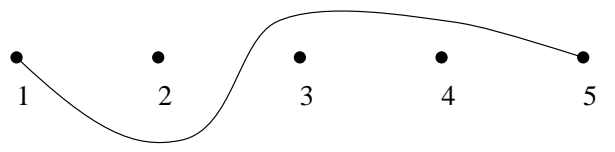

FiguRE 8.

From the resulting skeleton we compute the relation, as follows. We first explain the case when $\left[a_{i}, b_{i}\right]$ corresponds to a simple point, i.e. $b_{i}-a_{i}=1$. Then the skeleton is a path connecting two points.

Choose an arbitrary point on the path and 'pull' it down, breaking the path into two parts, which are connected in one end to $u_{0}$ and in the other to the two end points in $K$. 


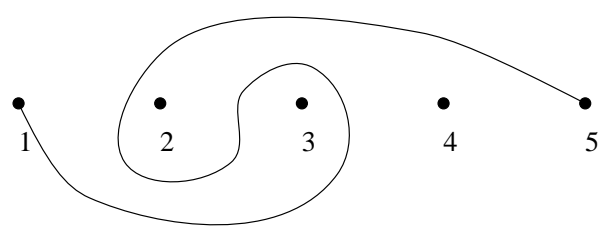

Figure 9 .

The loops associated to these two paths are elements in the group $\pi_{1}\left(D-K, u_{0}\right)$, and we call them $a_{1}$ and $a_{2}$. These are elements in $\pi_{1}\left(D-K, u_{0}\right)$, which commute in the fundamental group.

Figure 10 illustrates this procedure.

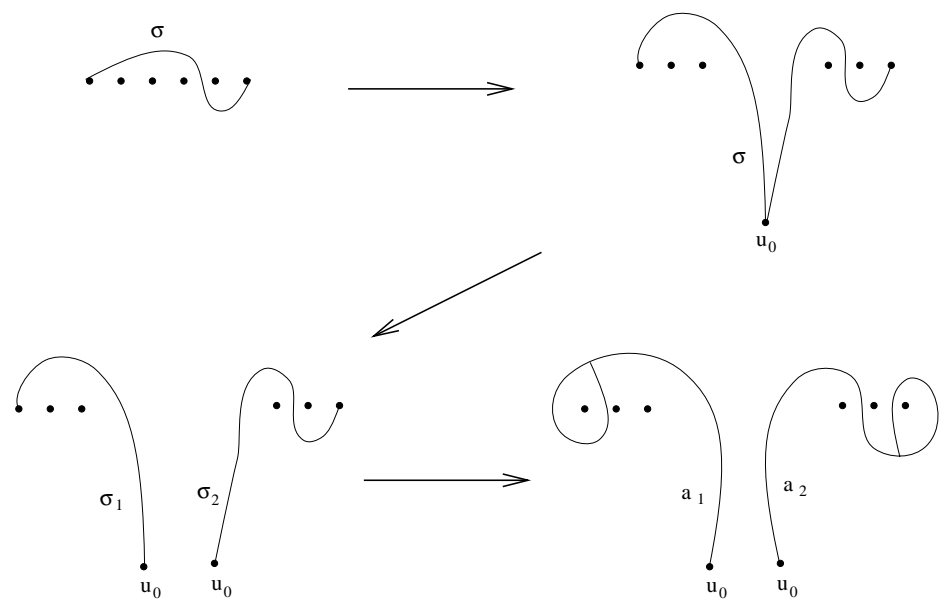

Figure 10.

Now we show how to write $a_{1}$ and $a_{2}$ as words on the generators $\left\{\Gamma_{1}, \cdots, \Gamma_{\ell}\right\}$ of $\pi_{1}\left(D-K, u_{0}\right)$. We begin with the generator corresponding to the end point of $a_{1}$ (or $a_{2}$ ), and conjugate it as we move along $a_{1}$ (or $a_{2}$ ) from its end point on $K$ to $u_{0}$ as follows: for every point $i \in K$ which we pass from above, we conjugate by $\Gamma_{i}$ when moving from left to right, and by $\Gamma_{i}^{-1}$ when moving from right to left.

For example, in the above figure,

$$
a_{1}=\Gamma_{3} \Gamma_{2} \Gamma_{1} \Gamma_{2}^{-1} \Gamma_{3}^{-1}, \quad a_{2}=\Gamma_{4}^{-1} \Gamma_{6} \Gamma_{4}
$$

and so the induced relation is:

$$
\Gamma_{3} \Gamma_{2} \Gamma_{1} \Gamma_{2}^{-1} \Gamma_{3}^{-1} \cdot \Gamma_{4}^{-1} \Gamma_{6} \Gamma_{4}=\Gamma_{4}^{-1} \Gamma_{6} \Gamma_{4} \cdot \Gamma_{3} \Gamma_{2} \Gamma_{1} \Gamma_{2}^{-1} \Gamma_{3}^{-1}
$$

One can check that the relation is independent of the point in which the path is broken. 
For a multiple intersection point we compute the elements in the group $\pi_{1}\left(D-K, u_{0}\right)$ in a similar way, but the induced relations are of the type

$$
a_{k} a_{k-1} \cdots a_{1}=a_{1} a_{k} \cdots a_{3} a_{2}=\cdots=a_{k-1} a_{k-2} \cdots a_{1} a_{k} .
$$

We choose an arbitrary point on the path and pull it down to $u_{0}$. For each of the $k$ end points of the skeleton, we generate the loop associated to the path from $u_{0}$ to that point, and translate this path to a word on $\Gamma_{1}, \ldots, \Gamma_{\ell}$ by the procedure given above.

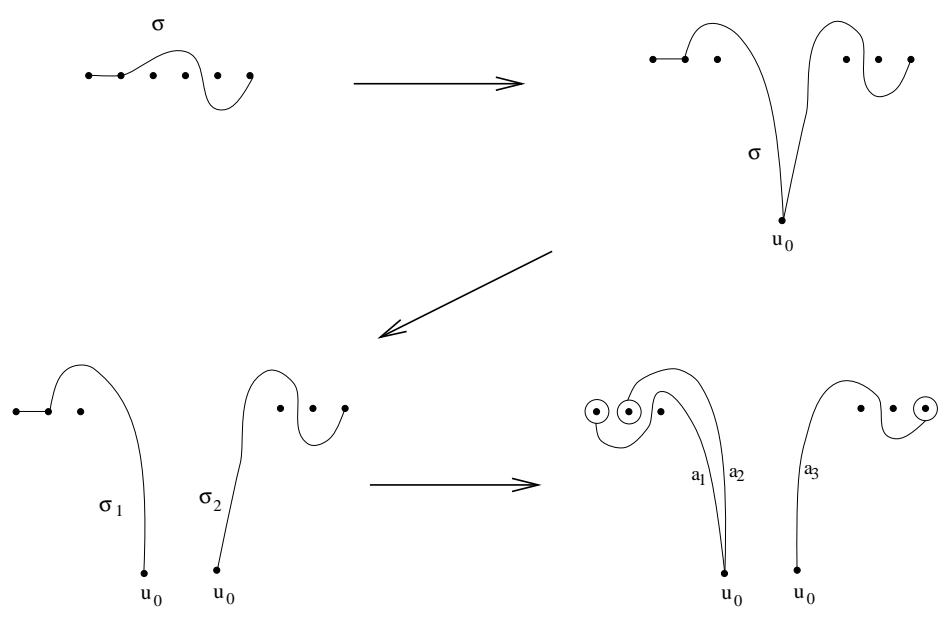

Figure 11.

In the example given in Figure 11, we have $a_{1}=\Gamma_{3} \Gamma_{1} \Gamma_{3}^{-1}, a_{2}=$ $\Gamma_{3} \Gamma_{2} \Gamma_{3}^{-1}$ and $a_{3}=\Gamma_{4}^{-1} \Gamma_{6} \Gamma_{4}$, so the relations are

$$
\begin{aligned}
\Gamma_{4}^{-1} \Gamma_{6} \Gamma_{4} \cdot \Gamma_{3} \Gamma_{2} \Gamma_{3}^{-1} \cdot \Gamma_{3} \Gamma_{1} \Gamma_{3}^{-1} & =\Gamma_{3} \Gamma_{1} \Gamma_{3}^{-1} \cdot \Gamma_{4}^{-1} \Gamma_{6} \Gamma_{4} \cdot \Gamma_{3} \Gamma_{2} \Gamma_{3}^{-1} \\
& =\Gamma_{3} \Gamma_{2} \Gamma_{3}^{-1} \cdot \Gamma_{3} \Gamma_{1} \Gamma_{3}^{-1} \cdot \Gamma_{4}^{-1} \Gamma_{6} \Gamma_{4} .
\end{aligned}
$$

Finally, the projective fundamental group $\pi_{1}\left(\mathbb{C P}^{2}-\mathbf{w}\right)$ is the quotient of the affine fundamental group $\pi_{1}\left(\mathbb{C}^{2}-\mathbf{w}\right)$ which we just computed, by the relation

$$
\Gamma_{\ell} \cdots \Gamma_{1}=1
$$

\section{RELATIONS AND ACTIONS ON WIRING DIAGRAMS}

Fix a signature $S=\left[2^{n_{2}} 3^{n_{3}} \ldots\right]$. Denote by $W_{S}$ the set of all lists of Lefschetz pairs with that given signature, for which the associated wiring diagram has the unique intersection property (defined in Subsection 2.3). Note that the number of lines and intersection points is determined by $S$ : there are $p=\sum n_{k}$ points, and the number of lines can be computed from Equation (11). 
In this section we introduce two equivalence relations and three actions on $W_{S}$ or its quotient sets. The motivation for the equivalence relations and the actions comes from the identities in the braid group and the isometries of the projective plane.

4.1. Disjoint intersection points. We say that two Lefschetz pairs $[a, b]$ and $[c, d]$ are disjoint if the correpsonding integral segments $[a, b]$ and $[c, d]$ are disjoint. If two adjacent pairs are disjoint, then the corresponding intersection points have no common lines.

We say that two lists of Lefschetz pairs $\mathbf{a}, \mathbf{b} \in W_{S}$ are equivalent, and denote $\mathbf{a} \equiv \mathbf{b}$, if it is possible to reach from $\mathbf{a}$ to $\mathbf{b}$ by switching adjacent disjoint pairs. This is an equivalence relation on $W_{S}$.

For example, the following two wiring diagrams are equivalent:

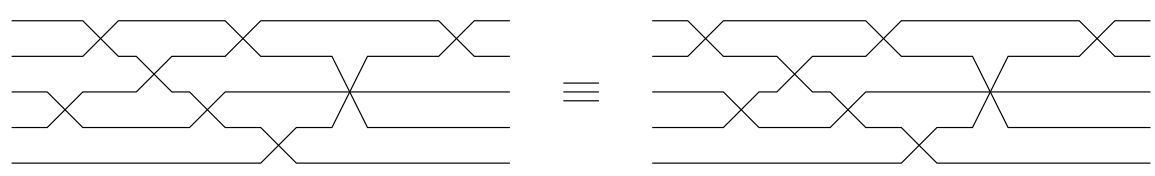

FiguRE 12.

Just as we have $\sigma_{i} \sigma_{j}=\sigma_{j} \sigma_{i}$ if $|j-i|>1$ for the standard generators of Artin's braid group, here too exchanging two disjoint points does not change the wiring diagram topologically, so we have

Proposition 4.1. If $\mathbf{a} \equiv \mathbf{a}^{\prime}$ then $\mathbf{a}$ and $\mathbf{a}^{\prime}$ have the same incidence lattice, and the same affine and projective fundamental groups.

4.2. Reflection. We define an action $\tau$ on $W_{S}$, motivated by a reflection of the plane by a line perpendicular to the guiding line:

Definition 4.2. For $\mathbf{a}=\left(\left[a_{1}, b_{1}\right],\left[a_{2}, b_{2}\right], \cdots,\left[a_{p}, b_{p}\right]\right) \in W_{S}$, set

$$
\tau(\mathbf{a}):=\left(\left[a_{p}, b_{p}\right],\left[a_{p-1}, b_{p-1}\right], \cdots,\left[a_{1}, b_{1}\right]\right) .
$$

Remark 4.3. If $\mathbf{a} \equiv \mathbf{b}$ then it is easy to see that $\tau(\mathbf{a}) \equiv \tau(\mathbf{b})$, so that $\tau$ is well-defined on $W_{S} / \equiv$.

Obviously $\tau$ does not change the wiring diagram topologically, so we have

Proposition 4.4. The wiring diagrams $\mathbf{a}$ and $\tau(\mathbf{a})$ have the same incidence lattice, and the same affine and projective fundamental groups. 
4.3. Rotation. The next action is related to rotation of the plane and its effect on wiring diagrams. Consider a smooth rotation of a line arrangement, when the guiding line is kept fixed. When we make a rotation, the projections of the intersection points slide along the guiding line. It may happen that two projections coincide (when two disjoint intersection points are one above the other; at that moment, the list of Lefschetz pairs induced from the wiring diagram is not defined). When we continue the rotation, the two projections switched places and we get a new wiring diagram, equivalent to the original one (in the sense of Subsection 4.1]).

A more radical and interesting change happens when one of the lines (the one numbered 1, when the rotation is clockwise) becomes perpendicular to the guiding line. Immediately before it happens, the projections of the intersection points on this line are consecutive, and immediately after the rotation their order is reversed. The local numbers of the lines from the left to these points are increased by one, and those from the right are decreased by one.

Example. The arrangement in Figure 13(a) was rotated clockwise, until the thick line is perpendicular to the guiding line, and then some. We get the arrangement given in Figure 13(b).

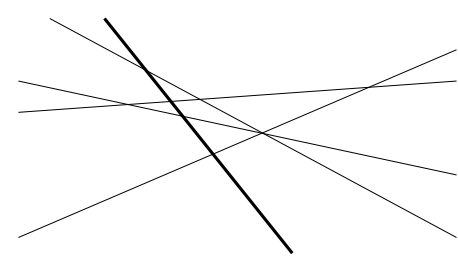

(a) Before $\mu$

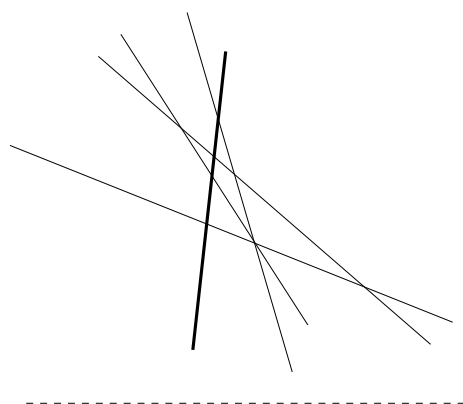

(b) After $\mu$

Figure 13.

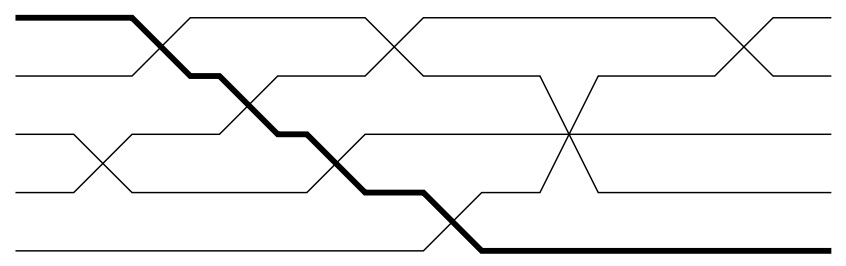

FiguRE 14. 
The wiring diagram corresponding to Figure 13(a) is given in Figure 14. The corresponding list of Lefschetz pairs is

$$
([4,5],[2,4],[1,2],[4,5],[2,3],[3,4],[4,5],[2,3]),
$$

which can be reordered to the following equivalent list:

$$
([4,5],[2,4],[4,5],[1,2],[2,3],[3,4],[4,5],[2,3])
$$

The wiring diagram corresponding to Figure 13(b) is given in Figure 15,

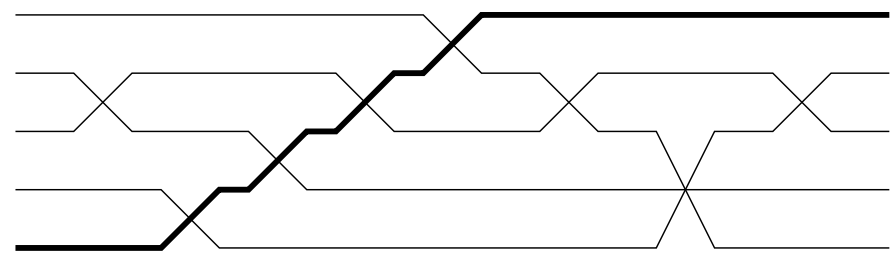

FigURE 15.

with the corresponding list of Lefschetz pairs being

$$
([3,4],[1,3],[3,4],[4,5],[3,4],[2,3],[1,2],[3,4]) .
$$

This definition for wiring diagrams induced by a line arrangement will now be generalized to arbitrary wiring diagrams with the unique intersection property.

Let $\mathbf{a}=\left(\left[a_{1}, b_{1}\right],\left[a_{2}, b_{2}\right], \cdots,\left[a_{p}, b_{p}\right]\right)$ be an element of $W_{S}$. We inductively construct a decomposition of the list of pairs in a into three disjoint sub-lists $L_{+}, L_{-}$and $L_{0}$. We view every $\left[a_{i}, b_{i}\right]$ as a permutation acting on the indices $\{1, \cdots, \ell\}$, where $\left[a_{i}, b_{i}\right]$ sends every $a_{i} \leq t \leq b_{i}$ to $a_{i}+b_{i}-t$, and leaves the other indices fixed.

Set $x=1$ and $L_{+}=L_{-}=L_{0}=\emptyset$. For each $i=1, \ldots, p$, act as follows. If $a_{i} \leq x \leq b_{i}$, add $\left[a_{i}, b_{i}\right]$ to the list $L_{0}$, and set $x=a_{i}+b_{i}-x$. If $x>b_{i}$, add $\left[a_{i}, b_{i}\right]$ to the list $L_{-}$, and if $x<a_{i}$, add $\left[a_{i}, b_{i}\right]$ to the list $L_{+}$. Continue to the next value of $i$.

During this procedure, the line numbered 1 at $\infty$ always carries the local index $x$. As a result, whenever $a_{i} \leq x \leq b_{i}$, we actually have $x=a_{i}$ (since otherwise the line with local number $a_{i}$ would have to intersect the first line at some point before, and again at point number $i$, contradicting the unique intersection property).

If follows that the intersection points which were combined as $L_{0}$ all lay on the first line, the points in the list $L_{+}$are above that line, and the ones in $L_{-}$are below it.

As an example, see Figure 14, where we have:

$L_{+}=([4,5],[2,4],[4,5]) ; L_{0}=([1,2],[2,3],[3,4],[4,5]) ; L_{-}=([2,3])$ 
It is now obvious that we can reorder $\mathbf{a}$ into an equivalent list $\mathbf{a}^{\prime}$, which can be written as an ordered union $\mathbf{a}^{\prime}=L_{+} \cup L_{0} \cup L_{-}$. Thus we can define an action $\mu$ as follows.

Definition 4.5. Let $\mathbf{a} \in W_{S}$ be a list of Lefschetz pairs,

$$
\mathbf{a}=\left(\left[a_{1}, b_{1}\right],\left[a_{2}, b_{2}\right], \cdots,\left[a_{p}, b_{p}\right]\right) .
$$

Decompose $\mathbf{a} \equiv L_{+} \cup L_{0} \cup L_{-}$as above, so that we can write:

$$
\begin{gathered}
L_{+}=\left(\left[a_{1}, b_{1}\right], \cdots,\left[a_{u}, b_{u}\right]\right), \\
L_{0}=\left(\left[a_{u+1}, b_{u+1}\right], \cdots,\left[a_{v}, b_{v}\right]\right), \\
L_{-}=\left(\left[a_{v+1}, b_{v+1}\right], \cdots,\left[a_{p}, b_{p}\right]\right) .
\end{gathered}
$$

where $1 \leq u<v \leq n$.

We substruct one from the indices in $L_{+}$, invert $L_{0}$ and add one to the indices in $L_{-} ; \mu(\mathbf{a})$ is defined by:

$$
\begin{aligned}
\mu(\mathbf{a}):=\left(\left[a_{1}-1, b_{1}-1\right], \cdots,\left[a_{u}-1, b_{u}-1\right],\right. \\
{\left[a_{v}, b_{v}\right],\left[a_{v-1}, b_{v-1}\right], \cdots,\left[a_{u+1}, b_{u+1}\right], } \\
{\left.\left[a_{v+1}+1, b_{v+1}+1\right], \cdots,\left[a_{p}+1, b_{p}+1\right]\right) . }
\end{aligned}
$$

In order for the definition to make sense, we must show that $\mu(\mathbf{a})$ is indeed a valid list of Lefschetz pairs (a list that can be induced from a wiring diagram with the unique intersection property), with the same signature.

This can be verified as follows. After reordering a to its equivalent form $\mathbf{a}^{\prime}=L_{+} \cup L_{0} \cup L_{-}$, break the two rays of line number 1 , and glue them back in exactly the opposite direction. Then rotate the middle section of this line until the order of the intersection points it carries is reversed. This transformation results in a new wiring diagram, which is easily seen to be the one corresponding to $\mu(\mathbf{a})$.

Remark 4.6. If $\mathbf{a}^{\prime} \equiv \mathbf{a}$, then $\mu\left(\mathbf{a}^{\prime}\right)=\mu(\mathbf{a})$.

Proof. The three sublists $L_{+}, L_{0}$ and $L_{-}$are the same for every $\mathbf{a}^{\prime} \equiv \mathbf{a}$. Therefore, $\mu\left(\mathbf{a}^{\prime}\right)$ is the same as well.

Since $\mu$ only shifts the numbers of lines, we have

Remark 4.7. The wiring diagrams a and $\mu(\mathbf{a})$ have the same incidence lattice.

Finally, we prove the following:

Proposition 4.8. Let $\mathbf{a}$ be a list of Lefschetz pairs, and let $\mathbf{w}$ be its associated wiring diagram. Let $\mathbf{w}_{\mu}$ be the wiring diagram associated to the list of Lefschetz pairs $\mu(\mathbf{a})$. Then

$$
\pi_{1}\left(\mathbb{C}^{2}-\mathbf{w}\right) \cong \pi_{1}\left(\mathbb{C}^{2}-\mathbf{w}_{\mu}\right)
$$


and

$$
\pi_{1}\left(\mathbb{C P}^{2}-\mathbf{w}\right) \cong \pi_{1}\left(\mathbb{C P}^{2}-\mathbf{w}_{\mu}\right)
$$

Proof. Since equivalent wiring diagrams have the same fundamental groups, we may reorder $\mathbf{w}$ so that all the intersection points on the first wire are consecutive (as explain in the definition of $\mu$ ). Then, by definition, the wiring diagram $\mathbf{w}_{\mu}$ is generated from $\mathbf{w}$ by breaking the first wire of $\mathbf{w}$ at the end points of its two rays. We can smoothly rotate them until we achieve the wiring diagram $\mathbf{w}_{\mu}$. Keeping the same angle of both rays along the rotation, the diagram is always topologically the same (even in $\mathbb{C P}^{2}$ ), and the result follows.

4.4. Beyond infinity. Our third action, denoted by $\sigma$, is well-defined on $W_{S}$ but not on $W_{S} / \equiv$.

Consider a wiring diagram associated to $\mathbf{a} \in W_{S}$. Recall that from the unique intersection property it follows that the order of the pseudolines at $\infty$ is the inverse of that at $-\infty$. Glue the wires together by identifying the two far edges of every wire. This gives an embedding of the wiring diagram on a Möbius strip. To recapture a, we simply cut the strip at $\infty$.

The action $\sigma$ is defined as follows: slide the point $\infty$ to the left (alternatively, rotate the strip to the right), until it crosses one intersection point. Then cut the strip at the new place of $\infty$.

The corresponding map on the set of lists of Lefschetz pairs is as follows (recall that the order of the lines is inverted in infinity). Let $\ell$ be the number of wires in the wiring diagram. Denote by $J=[1, \ell]$ the following permutation:

$$
J(i)=\ell+1-i, \quad 1 \leq i \leq \ell
$$

Definition 4.9. Let $\mathbf{a}=\left(\left[a_{1}, b_{1}\right],\left[a_{2}, b_{2}\right], \cdots,\left[a_{p}, b_{p}\right]\right) \in W_{S}$. Then we define

$$
\sigma(\mathbf{a}):=\left(\left[a_{2}, b_{2}\right], \cdots,\left[a_{p}, b_{p}\right],\left[J\left(b_{1}\right), J\left(a_{1}\right)\right]\right)
$$

To show that $\sigma(\mathbf{a})$ is again induced by a wiring diagram satisfying the unique intersection property, write $\mathbf{a}=\left(\pi_{1}, \cdots, \pi_{p}\right)$ where $\pi_{i}=\left[a_{i}, b_{i}\right]$ is the permutation that sends every $a_{i} \leq t \leq b_{i}$ to $a_{i}+b_{i}-t$, and leaves the other indices fixed. Note that the signature of $\left(\pi_{1}, \ldots, \pi_{p}\right)$ is given by the multiset of the sizes of supports of $\pi_{i}$, and, assuming the signature satisfies Equation (1),$\left(\pi_{1}, \ldots, \pi_{p}\right)$ corresponds to a wiring diagram with the unique intersection property iff $\pi_{1} \ldots \pi_{\ell}=J$. Now, $\sigma(\mathbf{a})=\left(\pi_{2}, \cdots, \pi_{p}, J \pi_{1} J^{-1}\right)$, and 


$$
\begin{aligned}
\pi_{2} \cdots \pi_{p} \cdot J \pi_{1} J^{-1} & =\pi_{1}^{-1}\left(\pi_{1} \pi_{2} \cdots \pi_{p}\right) J \pi_{1} J^{-1}= \\
& =\pi_{1}^{-1} J^{2} \pi_{1} J^{-1}=J
\end{aligned}
$$

since $J^{2}$ is the identity.

Examples. Consider the wiring diagram corresponding to

$$
\mathbf{a}=([1,2],[2,3],[1,2]),
$$

with signature $\left[2^{3}\right]$. Then $\sigma(\mathbf{a})=([2,3],[1,2],[2,3])$ and $\sigma^{2}(\mathbf{a})=\mathbf{a}$.

Here is a slightly more complicated example. In the following pair of line arrangements, the right one is obtained from the other by pushing the rightmost point "through infinity" until the two bold lines are parallel, then letting them intersect at the left side of the line arrangement.

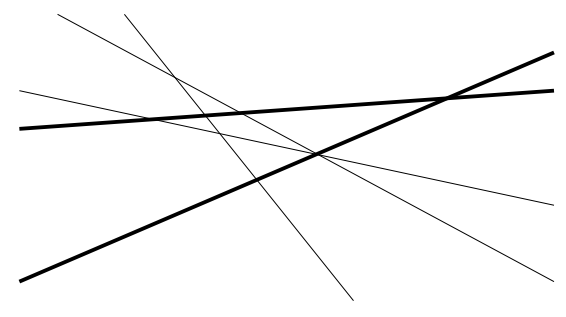

(a) Before $\sigma$

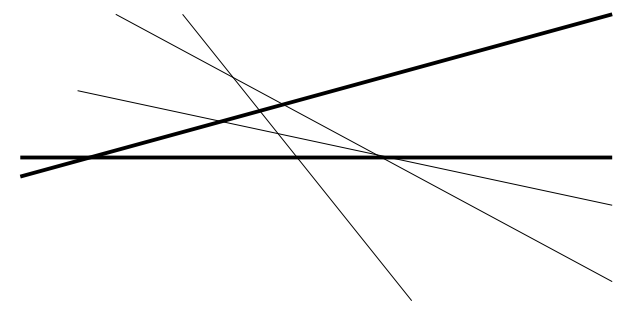

(b) After $\sigma$

Figure 16.

The wiring diagram corresponding to 16(a) is given in Figure 17. If

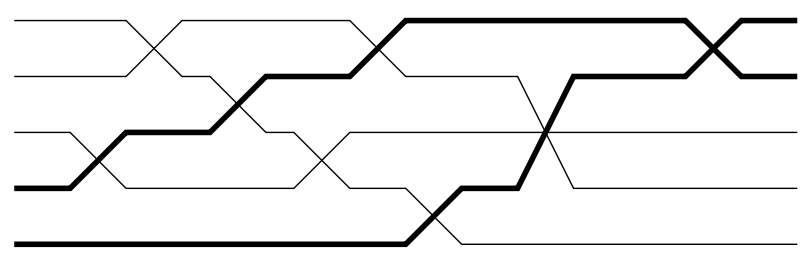

Figure 17.

we apply the above described action on the wiring diagram, we get the diagram in Figure 18. These figures demonstrate a simple point going 'through $\infty$ ', but the same procedure applies to a multiple intersection point as well.

As in previous actions, acting with $\sigma$ changes only the numeration of the points (and may also change the numbers of the lines, if the rightmost point involves the lowest line), so we have 


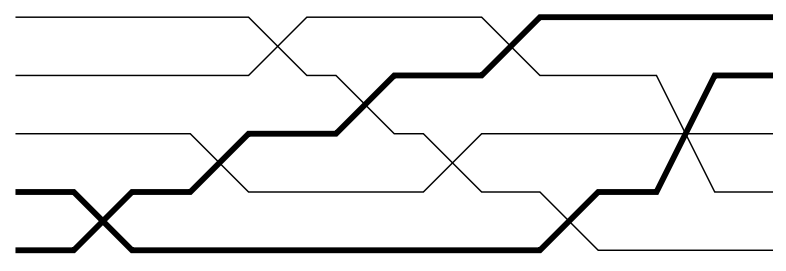

Figure 18.

Remark 4.10. For every $\mathbf{a} \in W_{S}$, a and $\sigma(\mathbf{a})$ have the same incidence lattice.

Next, we prove that $\sigma$ preserves the fundamental groups.

Theorem 4.11. For every wiring diagram $\mathbf{a} \in W_{S}$, a and $\sigma(\mathbf{a})$ have the same affine and projective fundamental groups.

Proof. We will in fact give an explicit isomorphism between the fundamental groups which were described in Section 3.

Let $\mathbf{a}=\left(\left[a_{1}, b_{1}\right], \cdots,\left[a_{p}, b_{p}\right]\right)$ be a list of Lefschetz pairs, and let $\mathbf{w}$ and $\mathbf{w}_{\sigma}$ be the wiring diagrams associated to $\mathbf{a}$ and $\sigma(\mathbf{a})$, respectively. Fix $a, s$ such that $[a, a+s]=\left[a_{1}, b_{1}\right]$.

Denote by $\Gamma_{1}, \ldots, \Gamma_{\ell}$ the geometric generators of $\pi_{1}\left(\mathbb{C}^{2}-\mathbf{w}\right)$, and by $\Gamma_{1}^{\prime}, \ldots, \Gamma_{\ell}^{\prime}$ the generators of $\pi_{1}\left(\mathbb{C}^{2}-\mathbf{w}_{\sigma}\right)$. We define $\phi: \pi_{1}\left(\mathbb{C}^{2}-\mathbf{w}\right) \rightarrow$ $\pi_{1}\left(\mathbb{C}^{2}-\mathbf{w}_{\sigma}\right)$ by

$$
\phi\left(\Gamma_{i}\right)=\left\{\begin{array}{cl}
\Gamma_{i}^{\prime} & 1 \leq i<a \\
\Gamma_{a}^{\prime-1} \cdots \Gamma_{a+s-1}^{\prime-1} \Gamma_{a+s}^{\prime} \Gamma_{a+s-1}^{\prime} \cdots \Gamma_{a}^{\prime} & i=a \\
\Gamma_{a}^{\prime-1} \cdots \Gamma_{a+s-2}^{\prime}{ }^{-1} \Gamma_{a+s-1}^{\prime} \Gamma_{a+s-2}^{\prime} \cdots \Gamma_{a}^{\prime} & i=a+1 \\
\cdots & \cdots \\
\Gamma_{a}^{\prime-1} \Gamma_{a+1}^{\prime} \Gamma_{a}^{\prime} & i=a+s-1 \\
\Gamma_{a}^{\prime} & i=a+s \\
\Gamma_{i}^{\prime} & a+s<i \leq \ell
\end{array}\right.
$$

This definition is motivated by Figure 19, which corresponds to an appropriate Hurwitz move $\mathrm{Hu}$.

In order to show that $\phi$ is well defined and is an isomorphism, we need to show that it carries the set of relations of $\pi_{1}\left(\mathbb{C}^{2}-\mathbf{w}\right)$ to the relations of $\pi_{1}\left(\mathbb{C}^{2}-\mathbf{w}_{\sigma}\right)$.

Recall that by definition

$$
\sigma(\mathbf{a})=\left(\left[a_{2}, b_{2}\right], \cdots,\left[a_{p}, b_{p}\right],\left[J\left(b_{1}\right), J\left(a_{1}\right)\right]\right) .
$$

We will first show that the relations in $\pi_{1}\left(\mathbb{C}^{2}-\mathbf{w}\right)$ associated to the points $\left[a_{2}, b_{2}\right], \ldots,\left[a_{p}, b_{p}\right]$ are equivalent to the relations in $\pi_{1}\left(\mathbb{C}^{2}-\mathbf{w}_{\sigma}\right)$ associated to the these points. 

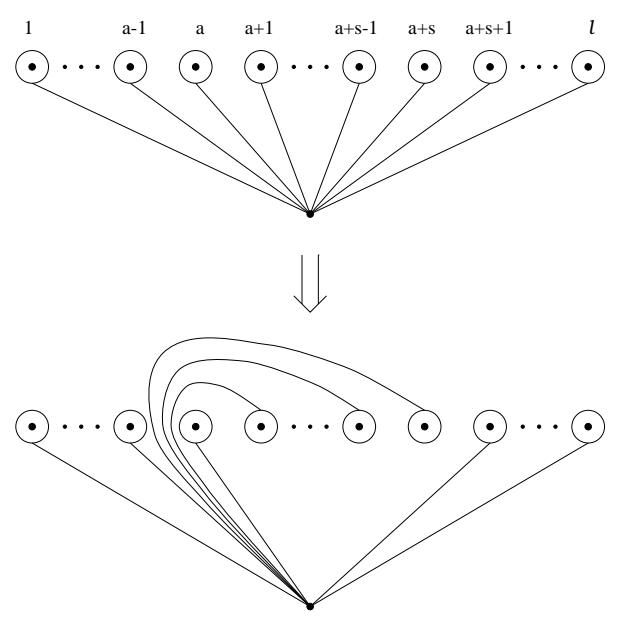

Figure 19.

Let $2 \leq j \leq p$. According to the algorithm in section 3 , the $j$ th skeleton of $\mathbf{a}$ is obtained by applying on the initial skeleton $\left[a_{j}, b_{j}\right]$ the halftwists corresponding to $\left[a_{j-1}, b_{j-1}\right]$, then to $\left[a_{j-2}, b_{j-2}\right]$, and so on, down to $\left[a_{1}, b_{1}\right]$. At the same time, the $(j-1)$ th skeleton of $\mathbf{w}_{\sigma}$ is obtained from the same initial skeleton, by applying $\left[a_{j-1}, b_{j-1}\right]$ down to $\left[a_{2}, b_{2}\right]$, without applying $\left[a_{1}, b_{1}\right]$. Therefore, in order to get the same skeleton in both cases, one has to act on the $j$ th skeleton of $\mathbf{w}$ by the inverse to the halftwist corresponds to $\left[a_{1}, b_{1}\right]$. The action of the Hurwitz move (and the map $\phi$ ) corresponds to this inverse action: it turns the local region in the disk clockwise (in the direction opposite to that of the halftwist's action). Therefore one gets the same skeletons, and hence equivalent relations in the presentation of the fundamental groups.

It remains to show that the relation induced by the skeleton corresponding to the first point, $\left[a_{1}, b_{1}\right]$, in $\mathbf{w}$, is equivalent to the relation induced by the skeleton corresponds to the last point, $\left[J\left(a_{1}\right), J\left(b_{1}\right)\right]$, of $\mathbf{w}_{\sigma}$.

According to the algorithm, the skeleton corresponding to $\left[a_{1}, b_{1}\right]$ for $\mathbf{w}$ is simply the straight segment between $a_{1}$ and $b_{1}$. The induced relation is (see GaTe ):

$$
\Gamma_{b_{1}} \cdots \Gamma_{a_{1}}=\Gamma_{b_{1}-1} \cdots \Gamma_{a_{1}} \Gamma_{b_{1}}=\cdots=\Gamma_{a_{1}} \Gamma_{b_{1}} \cdots \Gamma_{a_{1}+1} .
$$

For $\mathbf{w}_{\sigma}$, the skeleton is obtained by applying halftwists corresponding to the rest of the pairs, on the initial skeleton $\left[J\left(b_{1}\right), J\left(a_{1}\right)\right]$.

In the braid group, the action of all the $p$ halftwists of a list of Lefschetz pairs (with the unique intersection property) is equal to the action of the general halftwist $H([1, \ell])$, so the skeleton in this case can 
be obtained as follows: begin with the initial skeleton $\left[J\left(b_{1}\right), J\left(a_{1}\right)\right]$, apply $H([1, \ell])$ and then apply the inverse of the halftwist corresponding to $\left[a_{1}, b_{1}\right]$. Applying $H([1, \ell])$ on $\left[J\left(b_{1}\right), J\left(a_{1}\right)\right]$ yields the skeleton corresponding to the segment $\left[a_{1}, b_{1}\right]$. Then, applying the halftwist corresponds to $\left[a_{1}, b_{1}\right]$ will give the same result. Therefore we get the same skeleton, and hence equivalent sets of relations. This proves that the two affine groups are isomorphic.

Finally, compute that $\phi\left(\Gamma_{\ell} \ldots \Gamma_{1}\right)=\Gamma_{\ell}^{\prime} \ldots \Gamma_{1}^{\prime}$, so the relation defining the projective group of $\mathbf{w}$ transfers to the corresponding relation for $\mathbf{w}_{\sigma}$.

4.5. Going above and below. We define another relation on $W_{S}$, which can be motivated by the triple relation $\sigma_{i} \sigma_{i+1} \sigma_{i}=\sigma_{i+1} \sigma_{i} \sigma_{i+1}$ in the braid group. This relation corresponds to a line passing over a simple point. Surprisingly, it turns out that this change of the geometry of the arrangement, does not change the incidence lattice and fundamental groups.

We generalize this to a line passing over a multiple point. Let $t \geq 2$ denote the number of lines intersecting in a point, where the lines are locally numerated $c, \ldots, c+t-1$ (this point will be called here the central point). Now suppose another line crosses these $t$ lines, with all the intersection points adjacent to the central point. Let $c+i$ denote the index of this new line before the intersections, as in Figure 20. We claim that it does not matter if the new line crosses above or below that central point.
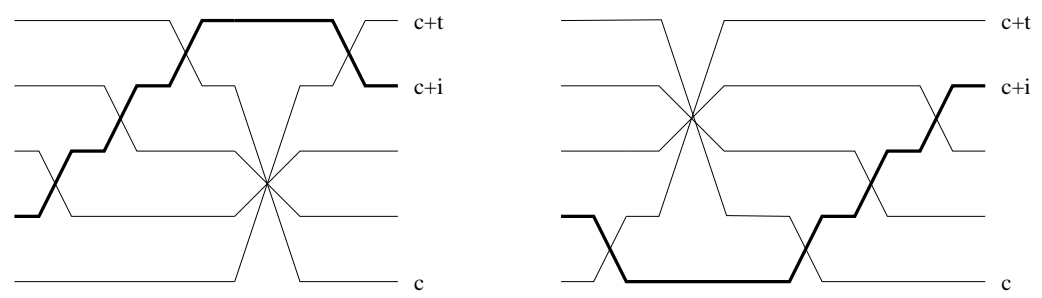

FiguRE 20.

In order to simplify the notation, we write $c+[a, b]$ instead of $[c+a, c+b]$, and more generally if $\mathbf{a}$ is a list of Lefschetz pairs, by $c+\mathbf{a}$ we mean adding $c$ to all the pairs in $\mathbf{a}$. In the situation described above we have $1 \leq c \leq c+i \leq c+t \leq \ell$, and the lists of Lefschetz pairs are $c+\triangle_{i}^{(t)}$ when the new line crosses from above, and $c+\nabla_{i}^{(t)}$ when it crosses from 
below, where we define

$$
\begin{aligned}
\triangle_{i}^{(t)}= & ([i, i+1], \cdots,[t-1, t],[0, t-1], \\
& {[t-1, t], \cdots,[t-i, t-i+1]), } \\
\nabla_{i}^{(t)}= & ([i-1, i], \cdots,[0,1],[1, t] \\
& {[0,1], \cdots,[t-1-i, t-i]) . }
\end{aligned}
$$

Definition 4.12. Let $\mathbf{a}, \mathbf{b} \in W_{S}$. We say that $\mathbf{a} \triangleq \mathbf{b}$, if there is a chain of replacements of $c+\triangle_{i}^{(t)}$ by $c+\nabla_{i}^{(t)}$, or vice versa, which goes from $\mathbf{a}$ to $\mathbf{b}$.

Since this relation changes only the positions of some intersection points, it obviously preserves the incidence lattice. We claim that $\triangleq$ also preserves the affine and projective fundamental groups.

Theorem 4.13. Let $\mathbf{a}, \mathbf{b} \in W_{S}$ be two lists of Lefschetz pairs such that $\mathbf{a} \triangleq \mathbf{b}$. Let $\mathbf{w}_{\mathbf{a}}$ and $\mathbf{w}_{\mathbf{b}}$ are the wiring diagrams associated to $\mathbf{a}$ and $\mathbf{b}$ respectively. Then $\mathbf{w}_{\mathbf{a}}$ and $\mathbf{w}_{\mathbf{b}}$ have the same affine and projective fundamental groups.

Proof. Let $\mathbf{a}$ and $\mathbf{b}$ be two lists of Lefschetz pairs, such that $\mathbf{a} \triangleq \mathbf{b}$. We have to show that $\pi_{1}\left(\mathbb{C}^{2}-\mathbf{w}_{\mathbf{a}}\right) \cong \pi_{1}\left(\mathbb{C}^{2}-\mathbf{w}_{\mathbf{b}}\right)$. It is obviously enough to assume the only difference between $\mathbf{a}$ and $\mathbf{b}$ is one replacement of $c+\triangle_{t}^{(i)}$ by $c+\nabla_{t}^{(i)}$, and by applying $\sigma$ we may assume that this replacement happens in the first $t+1$ pairs of each list. Then we have

$$
\mathbf{a}=\left(c+\triangle_{i}^{(t)},\left[a_{t+2}, b_{t+2}\right], \cdots,\left[a_{p}, b_{p}\right]\right),
$$

and

$$
\mathbf{b}=\left(c+\nabla_{i}^{(t)},\left[a_{t+2}, b_{t+2}\right], \cdots,\left[a_{p}, b_{p}\right]\right) .
$$

We will compute the presentations of $\pi_{1}\left(\mathbb{C}^{2}-\mathbf{w}_{\mathbf{a}}\right)$ and $\pi_{1}\left(\mathbb{C}^{2}-\mathbf{w}_{\mathbf{b}}\right)$ using the algorithm given in Section 3 , where $\left\{\Gamma_{1}, \Gamma_{2}, \ldots, \Gamma_{\ell}\right\}$ are the generators for the first group, and $\left\{\Gamma_{1}^{\prime}, \Gamma_{2}^{\prime}, \ldots, \Gamma_{\ell}^{\prime}\right\}$ for the second. We will show that the map $\Gamma_{j} \mapsto \Gamma_{j}^{\prime}$ is an isomorphism between the two groups.

We first show that the relations associated to the intersection points $\left[a_{j}, b_{j}\right]$ for $t+1<j \leq \ell$ are the same for both diagrams. According to the algorithm in section 3 , the $j$ th skeleton of $\mathbf{w}_{\mathbf{a}}$ is obtained by applying on the initial skeleton, associated to the pair $\left[a_{j}, b_{j}\right]$, the composition of the following halftwists: first, those associated to the pairs $\left[a_{k}, b_{k}\right], k=j-1, \ldots, t+2$, and then the $t+1$ halftwists associated to the pairs in $c+\triangle_{i}^{(t)}$. At the same time, the $j$ th skeleton of $\mathbf{w}_{\mathbf{b}}$ is obtained 
by applying on the same initial skeleton the same $j-(t+1)$ halftwists, and then the $t+1$ halftwists associated to the pairs in $c+\nabla_{i}^{(t)}$.

Since in the braid group the composition of the halftwists associated to the pairs in $c+\triangle_{i}^{(t)}$ is equal to the composition of the halftwists associated to the pairs in $c+\nabla_{i}^{(t)}$ (they both equal to the general halftwist $H([c, c+t]))$, we get the same final $j$ th skeleton for $\mathbf{w}_{\mathbf{a}}$ and for $\mathbf{w}_{\mathbf{b}}$. In particular, we get the same induced relations in both cases.

It remains to show that the relations induced by the first $t+1$ intersection points of $\mathbf{w}_{\mathbf{a}}$ are equivalent to the relations induced by the first $t+1$ intersection points of $\mathbf{w}_{\mathbf{b}}$. We will compute the relations in both cases.

Proposition 4.14. The relations induced on the generators $\Gamma_{1}, \ldots, \Gamma_{\ell}$ by the points listed in $c+\triangle_{i}^{(t)}$ are

$$
\begin{aligned}
& {\left[\Gamma_{c+i}, \Gamma_{c+k}\right]=1, \quad 0 \leq k \leq t} \\
& \Gamma_{c+t} \cdots \cdots \Gamma_{c}= \\
& \quad=\Gamma_{c+t-1} \cdots \Gamma_{c} \cdot \Gamma_{c+t}= \\
& \quad=\cdots=\Gamma_{c} \cdot \Gamma_{c+t} \cdots \cdots \Gamma_{c+1} .
\end{aligned}
$$

Proof. There are three types of skeletons to consider, corresponding to point before the central point, points after the central point, and the central point itself.

1. First let $1 \leq j \leq t-i$, so that the point is $c+[i+j-1, i+j]$. We need to apply the halftwists corresponding to the pairs $c+[i+k-1, i+k]$, $k=j-1, \ldots, 1$, on the initial skeleton which corresponds to $c+$ $[i+j-1, i+j]$. The resulting skeleton is given in Figure 21.

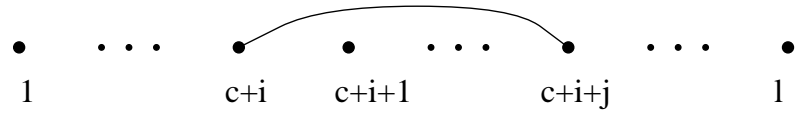

Figure 21.

This skeleton induces the following relation:

$$
\begin{aligned}
& \Gamma_{c+i} \cdot \Gamma_{c+i+1}^{-1} \cdots \Gamma_{c+i+j-1}^{-1} \Gamma_{c+i+j} \Gamma_{c+i+j-1} \cdots \Gamma_{c+i+1}= \\
& =\Gamma_{c+i+1}^{-1} \cdots \Gamma_{c+i+j-1}^{-1} \Gamma_{c+i+j} \Gamma_{c+i+j-1} \cdots \Gamma_{c+i+1} \cdot \Gamma_{c+i} .
\end{aligned}
$$

Induction on $j$ now proves Equation (2) for $i<k \leq t$.

2. We compute the relations for the other points, and then get back to the central point. Let $t-i+2 \leq j \leq t+1$, and consider the point $c+[2 t-i-j+1,2 t-i-j+2]$ which comes after the central point. To obtain the skeleton, we begin with the initial skeleton, given 
in Figure 22. We apply the halftwists corresponding to the pairs $c+$ $[2 t-i-k+1,2 t-i-k+2], k=j-1, \cdots, t-i+2$, obtaining the skeleton given in Figure 23; then the halftwist corresponding to the central point $[c, c+t-1]$, obtaining the skeleton in Figure 24, and finally the halftwists corresponding to the pairs $c+[i+(k-1), i+k]$, $k=t-i, \cdots, 1$, obtaining the final skeleton of Figure 25.

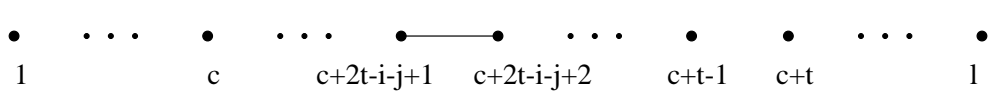

Figure 22.

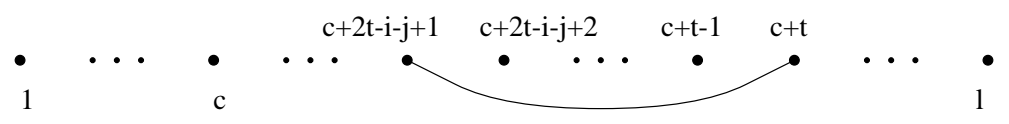

FigURE 23.

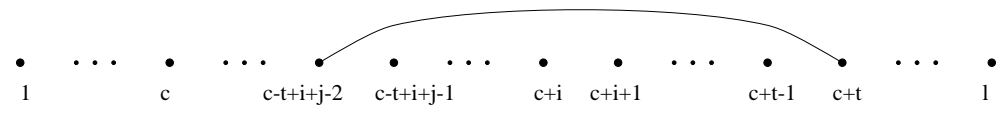

FiguRe 24.

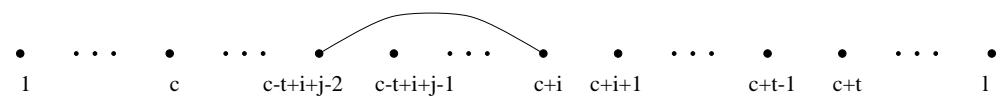

Figure 25.

The relation induced by this skeleton is

$$
\begin{gathered}
\Gamma_{c+i} \cdot \Gamma_{c+i-1} \cdots \Gamma_{c-t+i+j-1} \Gamma_{c-t+i+j-2} \Gamma_{c-t+i+j-1}^{-1} \cdots \Gamma_{c+i-1}^{-1}= \\
\Gamma_{c+i-1} \cdots \Gamma_{c-t+i+j-1} \Gamma_{c-t+i+j-2} \Gamma_{c-t+i+j-1}^{-1} \cdots \Gamma_{c+i-1}^{-1} \cdot \Gamma_{c+i} .
\end{gathered}
$$

Again by induction on $j$, we obtain the relations (2) for $0 \leq k<i$. Since (2) trivially holds for $k=i$, this set of relations is proved.

3. The central skeleton is obtained by applying the halftwists correspond to the pairs $c+[i+k-1, i+k], k=t-i, \ldots, 1$ on the initial skeleton corresponding to $[c, c+t-1]$, which is given in Figure 26. The resulting skeleton is given in Figure 27.

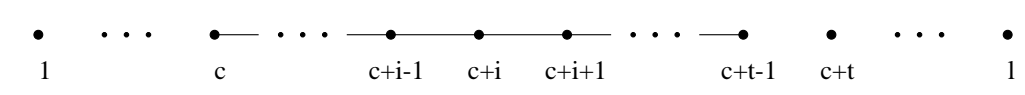

Figure 26. 


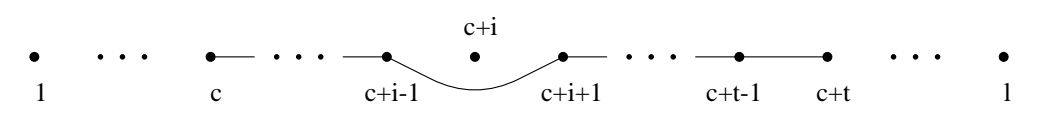

FiguRE 27.

The relation induced by this skeleton is

$$
\begin{gathered}
\Gamma_{c+t} \cdots \Gamma_{c+i+1} \cdot \Gamma_{c+i-1} \cdots \Gamma_{c}= \\
=\Gamma_{c+t-1} \cdots \Gamma_{c+i+1} \cdot \Gamma_{c+i-1} \cdots \Gamma_{c} \cdot \Gamma_{c+t}= \\
=\cdots=\Gamma_{c} \cdot \Gamma_{c+t} \cdots \cdots \Gamma_{c+i+1} \cdot \Gamma_{c+i-1} \cdots \Gamma_{c+1} .
\end{gathered}
$$

which is Equation (3) since by (2) $\Gamma_{c+i}$ commutes with all the generators appearing in this relation.

Next, we compute the corresponding relations induced by the skeletons correspond to the first $t+1$ intersection points of $\mathbf{w}_{\mathbf{b}}$.

Proposition 4.15. The relations induced on the generators $\Gamma_{1}, \ldots, \Gamma_{\ell}$ by the points listed in $c+\nabla_{i}^{(t)}$ are

$$
\begin{aligned}
& {\left[\Gamma_{c+i}^{\prime}, \Gamma_{c+k}^{\prime}\right]=1, \quad 0 \leq k \leq t} \\
& \Gamma_{c+t}^{\prime} \cdots \cdots \Gamma_{c}^{\prime}= \\
& \quad=\Gamma_{c+t-1}^{\prime} \cdots \cdots \Gamma_{c}^{\prime} \cdot \Gamma_{c+t}^{\prime}= \\
& \quad=\cdots=\Gamma_{c}^{\prime} \cdot \Gamma_{c+t}^{\prime} \cdots \cdots \Gamma_{c+1}^{\prime} .
\end{aligned}
$$

Proof. Again we treat the three classes of points separately.

1. Let $1 \leq j \leq i$. The $j$ th skeleton of $\mathbf{w}_{\mathbf{b}}$ is obtained by applying the halftwists corresponding to the pairs $c+[i-k, i-k+1], k=j-$ $1, \ldots, 1$ on the initial skeleton induced by the pair $c+[i-j, i-j+1]$. The resulting skeleton is given in Figure 28 .

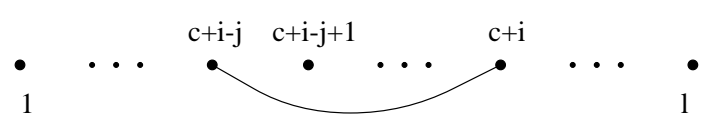

FiguRE 28.

This skeleton induces the relation $\Gamma_{c+i-j}^{\prime} \Gamma_{c+i}^{\prime}=\Gamma_{c+i}^{\prime} \Gamma_{c+i-j}^{\prime}$, and these are the cases $0 \leq k<i$ of Equation (雨).

2. Let $i+2 \leq j \leq t+1$. For the $j$ th skeleton we begin with the initial skeleton corresponding to $c+[i-j, i-j+1]$ (see Figure 29), apply the halftwists correspond to the pairs $c+[i-k, i-k+1]$, $k=j-1, \cdots, i-1$ to get the skeleton of Figure 30, then apply the central halftwist, corresponding to $[c+1, c+t]$, and get the skeleton of Figure 31, and finally apply the halftwists correspond to the pairs $c+$ 
$[i-k, i-k+1], k=i-1, \cdots, 1$, and get the final skeleton, presented in Figure 32 .

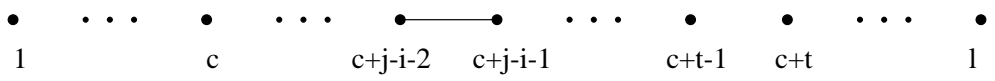

FiguRE 29.

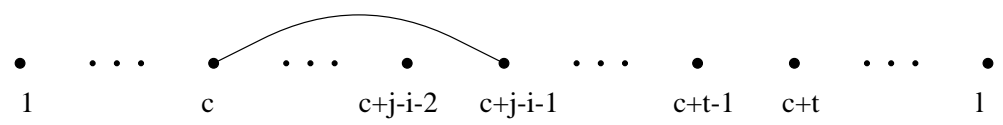

Figure 30.

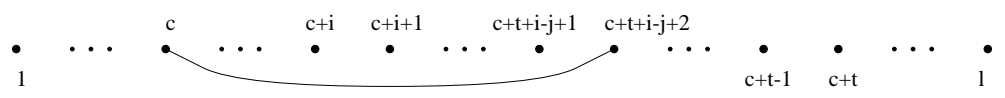

Figure 31.

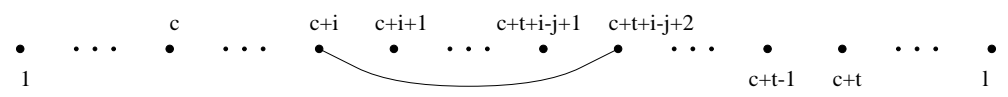

FigURE 32.

This skeleton induces the relation

$$
\Gamma_{c+i}^{\prime} \Gamma_{c+t+i-j+2}^{\prime}=\Gamma_{c+t+i-j+2}^{\prime} \Gamma_{c+i}^{\prime},
$$

and ranging over the possible values of $j$ we obtain the relation of Equation (4) for $i<k \leq t$.

3. The central skeleton is obtained by applying the halftwists correspond to the pairs $c+[i-k, i-k+1], k=i-1, \ldots, 1$ to the initial skeleton induced by the pair $[c+1, c+t]$, which is given in Figure 33 .

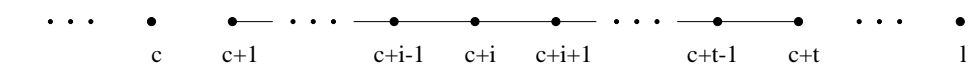

FiguRE 33.

The resulting skeleton is given in Figure 34 .

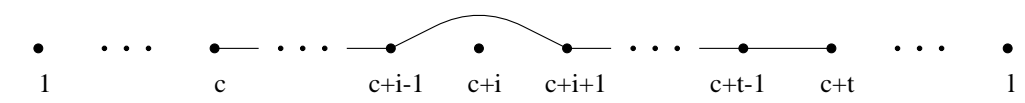

Figure 34. 
The corresponding induced relations are

$$
\begin{aligned}
& \Gamma_{c+i}^{\prime}{ }^{-1} \Gamma_{c+t}^{\prime} \Gamma_{c+i}^{\prime} \cdots \cdots \Gamma_{c+i}^{\prime}{ }^{-1} \Gamma_{c+i+1}^{\prime} \Gamma_{c+i}^{\prime} \cdot \Gamma_{c+i-1}^{\prime} \cdots \cdots \Gamma_{c}^{\prime}= \\
& \quad=\Gamma_{c+i}^{\prime}{ }^{-1} \Gamma_{c+t-1}^{\prime} \Gamma_{c+i}^{\prime} \cdots \cdots \Gamma_{c+i}^{\prime}{ }^{-1} \Gamma_{c+i+1}^{\prime} \Gamma_{c+i}^{\prime} \cdot \Gamma_{c+i-1}^{\prime} \cdots \cdots \Gamma_{c}^{\prime} \cdot \Gamma_{c+i}^{\prime}{ }^{-1} \Gamma_{c+t}^{\prime} \Gamma_{c+i}^{\prime}= \\
& \quad=\cdots=\Gamma_{c}^{\prime} \cdot \Gamma_{c+i}^{\prime}{ }^{-1} \Gamma_{c+t}^{\prime} \Gamma_{c+i}^{\prime} \cdots \cdots \Gamma_{c+i}^{\prime}{ }^{-1} \Gamma_{c+i+1}^{\prime} \Gamma_{c+i}^{\prime} \cdot \Gamma_{c+i-1}^{\prime} \cdots \cdots \Gamma_{c+1}^{\prime} .
\end{aligned}
$$

Using the relations (卧) we can simplify this to obtain relation (5).

Comparing the two propositions we discover the same set of relations, so the affine groups are shown to be isomorphic.

Since our isomorphism is $\Gamma_{j} \mapsto \Gamma_{j}^{\prime}$, the projective relation $\Gamma_{\ell} \cdots \Gamma_{1}=$ 1 goes to $\Gamma_{\ell}^{\prime} \cdots \Gamma_{1}^{\prime}=1$, so we have also proved that $\pi_{1}\left(\mathbb{C P}^{2}-\mathbf{w}_{\mathbf{a}}\right) \cong$ $\pi_{1}\left(\mathbb{C P}^{2}-\mathbf{w}_{\mathbf{b}}\right)$.

\section{Connections Between the aCtions}

In this section we point out two group actions induced by $\sigma, \tau$ and $\mu$. Note that it is meaningless to ask what is the group generated by $\sigma$ and $\mu$, since $\mu$ is defined only up to equivalence, and $\sigma$ is not well defined on equivalence classes. Let $D_{n}$ denote the dihedral group of order $2 n$.

Lemma 5.1. Let $S$ be a signature on $\ell$ pseudolines. Then $\mu$ and $\tau$ induce an action of the dihedral group $D_{2 \ell}$ on the set $W_{S} / \equiv$. In particular $\mu^{2 \ell}=1, \tau^{2}=1$, and $\tau \mu \tau=\mu^{-1}$ on this set.

Proof. $\tau$ inverts the order of the pairs, so that $\tau^{2}$ is the identity even on $W_{S}$.

By the geometric definition, each application of $\mu$ consists of inverting the direction of one pseudoline. $\mu^{\ell}(\mathbf{a})$ is a wiring diagram which is equivalent to a rotated by $180^{\circ}$. In particular, $\mu^{2 \ell}$ is the identity on $W_{S} / \equiv$.

It remains to check that $(\tau \mu)^{2}=\mathrm{Id}$. Let a be a list of Lefschetz pairs. As in the definition of $\mu$, we can decompose some $\mathbf{a}^{\prime} \equiv \mathbf{a}$ into three disjoint consecutive sublists: $\mathbf{a}^{\prime}=L_{+} \cup L_{0} \cup L_{-}$, where we write: $L_{+}=$ $\left(\left[a_{1}, b_{1}\right], \cdots,\left[a_{m}, b_{m}\right]\right), L_{0}=\left(\left[1, c_{1}\right],\left[c_{1}, c_{2}\right], \cdots,\left[c_{k-2}, c_{k-1}\right],\left[c_{k-1}, \ell\right]\right)$, and $L_{-}=\left(\left[d_{1}, e_{1}\right], \cdots,\left[d_{p}, e_{p}\right]\right)$. 
Now, compute:

$$
\begin{aligned}
\tau \mu \tau \mu\left(\mathbf{a}^{\prime}\right)= & \tau \mu \tau \mu\left(\left[a_{1}, b_{1}\right], \cdots,\left[a_{m}, b_{m}\right],\right. \\
& {\left[1, c_{1}\right],\left[c_{1}, c_{2}\right], \cdots,\left[c_{k-2}, c_{k-1}\right],\left[c_{k-1}, \ell\right], } \\
& {\left.\left[d_{1}, e_{1}\right], \cdots,\left[d_{p}, e_{p}\right]\right)=} \\
= & \tau \mu \tau\left(\left[a_{1}-1, b_{1}-1\right], \cdots,\left[a_{m}-1, b_{m}-1\right],\right. \\
& {\left[c_{k-1}, \ell\right],\left[c_{k-2}, c_{k-1}\right], \cdots,\left[c_{1}, c_{2}\right],\left[1, c_{1}\right], } \\
& {\left.\left[d_{1}+1, e_{1}+1\right], \cdots,\left[d_{p}+1, e_{p}+1\right]\right)=} \\
= & \tau \mu\left(\left[d_{p}+1, e_{p}+1\right], \cdots,\left[d_{1}+1, e_{1}+1\right],\right. \\
& {\left[1, c_{1}\right],\left[c_{1}, c_{2}\right], \cdots,\left[c_{k-2}, c_{k-1}\right],\left[c_{k-1}, \ell\right], } \\
& {\left.\left[a_{m}-1, b_{m}-1\right], \cdots,\left[a_{1}-1, b_{1}-1\right]\right)=} \\
\tau & \left(\left[d_{p}, e_{p}\right], \cdots,\left[d_{1}, e_{1}\right],\right. \\
& {\left[c_{k-1}, \ell\right],\left[c_{k-2}, c_{k-1}\right], \cdots,\left[c_{1}, c_{2}\right],\left[1, c_{1}\right], } \\
& {\left.\left[a_{m}, b_{m}\right], \cdots,\left[a_{1}, b_{1}\right]\right)=} \\
= & \left(\left[a_{1}, b_{1}\right], \cdots,\left[a_{m}, b_{m}\right],\right. \\
& {\left[1, c_{1}\right],\left[c_{1}, c_{2}\right], \cdots,\left[c_{k-2}, c_{k-1}\right],\left[c_{k-1}, \ell\right], } \\
& {\left.\left[d_{1}, e_{1}\right], \cdots,\left[d_{p}, e_{p}\right]\right)=\mathbf{a}^{\prime} . }
\end{aligned}
$$

Lemma 5.2. Assume that the signature $S$ has $p$ intersection points. Then $\sigma$ and $\tau$ induce an action of the dihedral group $D_{2 p}$ on $W_{S}$. In particular $\sigma^{2 p}=1, \tau^{2}=1$ and $\tau \sigma \tau^{-1}=\sigma^{-1}$.

Proof. Let $\mathbf{a}=\left(\left[a_{1}, b_{1}\right],\left[a_{2}, b_{2}\right], \cdots,\left[a_{p}, b_{p}\right]\right)$. By definition,

$$
\sigma^{p}(\mathbf{a})=\left(\left[J\left(b_{1}\right), J\left(a_{1}\right)\right],\left[J\left(b_{2}\right), J\left(a_{2}\right)\right], \cdots,\left[J\left(b_{p}\right), J\left(a_{p}\right)\right]\right)
$$

so that $\sigma^{2 p}=1$.

As was shown in the last lemma, the order of $\tau$ is 2 . Therefore, in order to show that $\langle\sigma, \tau\rangle$ is dihedral of order $4 p$, it remains to verify that $\sigma \tau \sigma \tau=$ Id. Indeed,

$$
\begin{aligned}
\sigma \tau \sigma \tau(\mathbf{a}) & =\sigma \tau \sigma \tau\left(\left[a_{1}, b_{1}\right],\left[a_{2}, b_{2}\right], \cdots,\left[a_{p}, b_{p}\right]\right) \\
& =\sigma \tau \sigma\left(\left[a_{p}, b_{p}\right],\left[a_{p-1}, b_{p-1}\right], \cdots,\left[a_{1}, b_{1}\right]\right) \\
& =\sigma \tau\left(\left[a_{p-1}, b_{p-1}\right],\left[a_{p-2}, b_{p-2}\right], \cdots,\left[a_{1}, b_{1}\right],\left[J\left(b_{p}\right), J\left(a_{p}\right)\right]\right) \\
& =\sigma\left(\left[J\left(b_{p}\right), J\left(a_{p}\right)\right],\left[a_{1}, b_{1}\right], \cdots,\left[a_{p-2}, b_{p-2}\right],\left[a_{p-1}, b_{p-1}\right]\right) \\
& =\left(\left[a_{1}, b_{1}\right], \cdots,\left[a_{p-2}, b_{p-2}\right],\left[a_{p-1}, b_{p-1}\right],\left[J J\left(a_{p}\right), J J\left(b_{p}\right)\right]\right) \\
& =\left(\left[a_{1}, b_{1}\right], \cdots,\left[a_{p-2}, b_{p-2}\right],\left[a_{p-1}, b_{p-1}\right],\left[a_{p}, b_{p}\right]\right)=\mathbf{a} .
\end{aligned}
$$


Actually, $\langle\sigma\rangle$ can have orbits of size smaller than $2 p$, as shown by the following example.

Example. Let $S$ be the signature $\left[2^{3} 3^{6}\right]$ of an arrangement of 7 pseudolines and 9 intersection points (Kelly-Moser's configuration [KM]). Let

$$
\mathbf{a}=([3,5],[1,3],[5,6],[3,5],[5,7],[2,3],[3,5],[1,3],[5,6]) .
$$

One can check that $\sigma^{6}(\mathbf{a})=\mathbf{a}$. This diagram has orbit of size 12 under the action of $\langle\sigma, \tau\rangle$.

In the proof of Lemma 5.2 we have seen that $\sigma^{p}$ is a reflection with respect to a line parallel to the rays of our pseudolines. In particular $\sigma^{p}$ is well defined on $W_{S} / \equiv$ (even though $\sigma$ is not). Similarly, we have shown in Lemma 5.1 that $\mu^{\ell}$ corresponds to a rotation of the diagram by $180^{\circ}$, so their composition is a vertical reflection which corresponds to $\tau$.

Corollary 5.3. As actions of the set $W_{S} / \equiv$, we have that

$$
\tau=\sigma^{p} \mu^{\ell}
$$

As a summary, we now list the number of classes under various sets of actions and relations. Consider the signature $S=\left[2^{13} 3^{3} 4^{1}\right]$ (on 8 lines).

The table below gives the number of equivalence classes of $W_{S} / \equiv$ under the various relations. For example, the forth line tells us that if two wiring diagrams are considered similar whenever there a chain of moving from a digram to an equivalent diagram (under $\equiv$ or $\triangleq$ ), or of application of $\mu$, then there are 6104 similarity classes. 


\begin{tabular}{|cccc||c|}
\hline$\sigma$ & $\tau$ & $\mu$ & $\triangleq$ & Number of classes \\
\hline- & - & - & - & 354880 \\
- & - & - & + & 114379 \\
- & - & + & - & 22180 \\
- & - & + & + & 6104 \\
- & + & - & - & 177440 \\
- & + & - & + & 54539 \\
- & + & + & - & 11090 \\
- & + & + & + & 3076 \\
+ & - & - & - & 5060 \\
+ & - & - & + & 772 \\
+ & - & + & - & 116 \\
+ & - & + & + & 22 \\
+ & + & - & - & 2558 \\
+ & + & - & + & 398 \\
+ & + & + & - & 116 \\
+ & + & + & + & 22 \\
\hline
\end{tabular}

We also computed the incidence lattices and the fundamental groups for each of the 22 similarity classes (when all the relations and actions are considered). There are 5 different lattices, and the classes which fall under the same lattice turn out to have the same fundamental groups.

\section{REFERENCES}

[CS] Cohen, D. C. and Suciu, A. I., The braid monodromy of plane algebraic curves and hyperplane arrangements, Comment. Math. Helvetici 72, 285-315 (1997).

[GaTe] Garber, D. and Teicher, M., The fundamental group's structure of the complement of some configurations of real line arrangements, Complex Analysis and Algebraic Geometry, edited by T. Peternell and F.-O. Schreyer, de Gruyter, 173-223 (2000).

[GTV] Garber, D., Teicher, M. and Vishne, U., $\pi_{1}$-classification of real arrangements with up to eight lines, submitted.

[Go] Goodman, J. E., Proof of a conjecture of Burr, Grünbaum and Sloane, Discrete Math. 32, 27-35 (1980).

[GP] Goodman, J. E. and Pollack, R., Allowable sequences and ordered types in discrete and computational geometry, in: New trends in discrete and computational geometry, edited by J. Pach, Springer-Verlag, 103-134 (1993).

[Gr] Grünbaum, B., Arrangements and spreads, Amer. Math. Soc., Providence (1972).

[Hu] Hurwitz, A., Über Riemann'sche Flächen mit gegebenen verzweigungspunkten, Math. Ann. 39, 1-60 (1891).

$[\mathrm{KM}]$ Kelly, L. M. and Moser, W. O. J., On the number of ordinary lines determined by $n$ points, Canad. J. Math. 10, 210-219 (1958).

[Le] Levi, F., Die Teillung der projektiven ebene durch gerade oder pseudogerade, Der. Math.-Phys. Kl. Sächs Akad. Wiss. 78, 256-267 (1926). 
[MoTe] Moishezon, B. G. and Teicher, M., Braid Group Technique in Complex Geometry I, Line Arrangements in $\mathbb{C P}^{2}$, Contemporary Math. 78, 425-555 (1988).

[OrT] Orlik, P. and Terao, H., Arrangements of Hyperplanes, Grundlehren 300, Springer-Verlag (1992).

[Ri] Ringel, G., Teilungen der ebene durch geraden oder topologische geraden, Math. Zeitschrift 64, 79-102 (1956).

E-mail address: \{garber,teicher\}@macs.biu.ac.il

Dept. of Math. and CS, Bar-Ilan University, Ramat-Gan 52900, ISRAEL

E-mail address: vishne@math.huji.ac.il

Einstein Institute of Mathematics, Hebrew University, Jerusalem 91904, ISRAEL 